\title{
Emergence in Effective Field Theories
}

\author{
Jonathan Bain \\ Polytechnic Institute of New York University \\ 6 Metrotech Center, Brooklyn, NY 11201
}

\begin{abstract}
1. Introduction.
2. EFTs and the Elimination of Degrees of Freedom.

3. An Interpretation of EFTs.

4. Emergence in EFTs.

5. Other Notions of Emergence.

6. Conclusion.
\end{abstract}

Abstract. This essay considers the extent to which a concept of emergence can be associated with Effective Field Theories (EFTs). I suggest that such a concept can be characterized by microphysicalism and novelty underwritten by the elimination of degrees of freedom from a high-energy theory, and argue that this makes emergence in EFTs distinct from other concepts of emergence in physics that have appeared in the recent philosophical literature.

\section{Introduction}

An effective field theory (EFT) of a physical system is a description of the system at energies low, or distances large, compared to a given cutoff. EFTs are constructed via a process in which degrees of freedom are eliminated from a high-energy/short-distance theory. Formulating a concept of emergence for EFTs is important for at least two reasons. First, EFTs play essential roles in contemporary physics: many authors believe the Standard Model of particle physics is an EFT, and most if not all condensed matter systems can be described by EFTs. Second, the types of physical systems that can be described by EFTs have been associated with various concepts of emergence in the recent philosophical literature: Mainwood (2006) suggests that the "new emergentism" of condensed matter physicists (e.g., Anderson 1972, Laughlin and Pine 2000) can be characterized by microphysicalism and novelty underwritten by the physical mechanisms of spontaneous symmetry breaking and universality. Morrison (2012) similarly stresses the role of spontaneous symmetry breaking as essential to a concept of emergence, while Batterman (2011) focuses on universality. On the other hand, Wilson (2010) claims an appropriate concept of emergence should be based on the elimination of degrees of freedom from a theory in physics. I will suggest that while a concept of emergence appropriate for EFTs shares aspects of these views, it is distinct from them.

The plan of the essay is as follows. Section 2 reviews the steps involved in the construction of an EFT, section 3 offers an interpretation of EFTs from which section 4 extracts a concept of emergence based on the notions of microphysicalism and novelty. Finally, section 5 compares this concept with recent discussion of emergence in the philosophical literature.

\section{EFTs and the Elimination of Degrees of Freedom}

The concept of emergence I wish to associate with EFTs will ultimately be based on the elimination of degrees of freedom from a field theory in physics. I will take a degree of freedom associated with a theory to be a parameter that needs to be assigned a value in order to provide a dynamical state description of a physical system described by the theory. A dynamical state 
description is a description of the system at an instant in time that, in conjunction with an equation of motion, determines a future or a past state. Thus, for example, a dynamical state description of a free classical particle governed by a second-order partial differential equation of motion (Newton's second law, for instance) is specified by the values of its position and momentum. In three spatial dimensions, this amounts to 6 degrees of freedom. A dynamical state description of a free classical field $\phi(x)$ governed by a second-order partial differential equation of motion is specified by the values that $\phi(x)$ and its first derivative $\partial_{\mu} \phi(x)$ take at every point $x$ of spacetime, which amounts to an infinite number of degrees of freedom.

For some field theories, degrees of freedom associated with high energies (or short distances) can be eliminated in such a way that the result is an effective field theory that produces the same predictions as the original when restricted to low energies (large distances). One advantage of using the effective theory is that it makes calculations more tractable. Moreover, many quantum field theories can only be solved via perturbative expansions which contain divergent integrals at high energies. For these theories, the construction of a low-energy effective theory provides not just a practical way of avoiding these divergences, but a conceptual framework on which to build an interpretation of what these theories are telling us about the world. This construction proceeds in two steps:

(I) The high-energy degrees of freedom are identified and integrated out of the Lagrangian density representing the theory.

This first step assumes that the theory is encoded in a Lagrangian density $\mathcal{L}[\phi]$, which is a functional of a field variable $\phi(x) .{ }^{1}$ This means that $\mathcal{L}[\phi]$ depends on all the possible functional forms the field can take, each form $\phi(x)$ taking values at all spacetime points $x$. Each such form of $\phi(x)$ represents a possible field configuration of field values; i.e., a possible way the field could be spread over spacetime. To identify the high-energy degrees of freedom, one first choses an appropriate energy cutoff $\Lambda$ and then decomposes the field variable into high- and lowenergy parts, $\phi(x)=\phi_{H}(x)+\phi_{L}(x)$, where $\phi_{H}(x)$ and $\phi_{L}(x)$ are associated with momenta greater than and less than $\Lambda$, respectively. Once this is done, the high-energy degrees of freedom $\phi_{H}(x)$ are integrated out of the generating functional $Z$ constructed from $\mathcal{L}\left[\phi_{H}, \phi_{L}\right]$,

$$
Z=\int \mathcal{D} \phi_{L} \mathcal{D} \phi_{H} e^{i \int d^{4} x \mathcal{L}\left[\phi_{L}, \phi_{H}\right]}=\int \mathcal{D} \phi_{L} e^{i \int d^{4} x \mathcal{L}_{e f f}\left[\phi_{L}\right]}
$$

This functional integral is taken over all possible field configurations of the high-energy degrees of freedom $\phi_{H}(x)$. This literally eliminates these degrees of freedom from the Lagrangian density by replacing them with appropriate configurations of the remaining degrees of freedom (conceptually, in the same way a variable $y$ is eliminated from an algebraic equation $a x+b y=c$, by replacing it with an appropriate relation for the remaining variable: $y=(c-a x) / b)$. The result of this is an effective Lagrangian density $\mathcal{L}_{\text {eff }}\left[\phi_{L}\right]$ that depends only on the low-energy degrees of freedom $\phi_{L}(x)$.

\footnotetext{
${ }^{1}$ In general a Lagrangian density of a field theory $\mathcal{L}\left[\phi_{i}, \partial_{\mu} \phi_{i}\right], i=1 \ldots N, \mu=0,1,2,3$, is a functional of $N$ field variables $\phi_{i}(x)$ and their first (and possibily higher-order) derivatives. For the sake of exposition, I'll restrict attention to a single scalar field variable.
} 
Typically, the functional integral over $\phi_{H}(x)$ in (1) is not exactly solvable, and even when it is, it may result in an effective Langrangian density that contains non-local terms (in the sense of depending on more than one spacetime point). These problems are jointly addressed by the second step in the construction of an EFT:

(II) The effective Lagrangian density is expanded in a local operator expansion

$$
\mathcal{L}_{\text {eff }}=\mathcal{L}_{0}+\sum_{i} c_{i} \mathcal{O}_{i}
$$

where $\mathcal{L}_{0}$ can be taken to be the interaction-free Lagrangian density (for weak interactions), the $c_{i}$ are coupling constants, and the sum runs over all local operators $\mathcal{O}_{i}$ allowed by the symmetries of $\mathcal{L}$.

Steps (I) and (II) can be characterized in the following ways:

(i) First, the effective Lagrangian density is formally distinct from the high-energy Lagrangian density. To the extent that this entails that the Euler-Lagrange equations of motion of the effective theory are distinct from those of the high-energy theory, the low-energy degrees of freedom $\phi_{L}(x)$ are dynamically distinct from the original degrees of freedom $\phi(x){ }^{2}$

(ii) Second, while the local operator expansion in Step II can be viewed formally as an approximate perturbative solution to the path integral (1), one can argue that an effective Lagrangian density is not simply an approximation of a high-energy Lagrangian density. In many cases, the exact form of the high-energy Lagrangian density is unknown, but an effective Langrangian density can still be constructed. Such a "bottom-up" EFT is obtained by including in the local operator expansion (2) all terms consistent with the symmetries and interactions assumed to be relevant at the energy scale of interest. A "folk theorem" identified by Weinberg (1979, pg. 329) then justifies viewing such bottom-up EFTs as not simply approximations to a high-energy theory. ${ }^{3}$ This suggests that, even in the context of a "top-down" EFT for which a high-energy theory is known, the local operator expansion conceptually stands on its own.

(iii) Finally, the elimination of degrees of freedom in the construction of an EFT results from the imposition of a constraint (an energy, or minimum length, cut-off) directly on a Lagrangian density, as opposed to a set of equations of motion. Again, the result is a formally distinct effective Lagrangian density with a distinct set of equations of motion and a distinct set of dynamical variables.

\footnotetext{
${ }^{2}$ For a Lagrangian density $\mathcal{L}\left[\phi_{i}, \partial_{\mu} \phi_{i}\right], i=1 \ldots N$, the Euler-Lagrange equations of motion are defined by $\partial \mathcal{L} / \partial \phi_{i}-$ $\partial_{\mu}\left(\partial \mathcal{L} / \partial\left(\partial_{\mu} \phi_{i}\right)\right)=0$.

${ }^{3}$ The folk theorem states that "...if one writes down the most general possible Lagrangian, and then calculates matrix elements with this Lagrangian to any given order of perturbation theory, the result will simply be the most general possible $S$-matrix consistent with analyticity, perturbative unitarity, cluster decomposition, and the assumed symmetry principles" (Weinberg 1979, pg. 329).
} 


\section{An Interpretation of EFTs}

The fact that EFTs come in two flavors, top-down and bottom-up, and that only the former is explicitly associated with a high-energy theory, might initially give one pause in attempting to formulate a notion of emergence appropriate for EFTs. In particular, the concern might be that such a notion assumes a distinction between a theory that describes emergent phenomena and a second theory that describes phenomena from which the former emerge; and such a distinction can only be made in the case of a top-down EFT. But this objection is easily blunted: Nothing in the construction of a bottom-up EFT precludes us from assuming that an associated highenergy theory exists; rather, the working assumption is simply that we do not know the form this high-energy theory takes. (A high-energy theory in this context need only be a theory that describes phenomena at an energy scale above that associated with an EFT; i.e., it need not be a Grand Unified Theory applicable to all energy scales in toto.) Moreover, even in the top-down context, the EFT does not completely determine the form of the high-energy theory: for a given high-energy theory, more than one top-down EFT can be constructed.

These considerations suggest the following interpretation of EFTs, both top-down and bottomup:

(a) Failure of law-like deducibility. If we understand the laws of a theory encoded in a Lagrangian density to be its Euler-Lagrange equations of motion, then the phenomena described by an EFT are not deducible consequences of the laws of a high-energy theory.

(b) Ontological distinctness. The degrees of freedom of an EFT characterize physical systems that are ontologically distinct from physical systems characterized by the degrees of freedom of a high-energy theory.

(c) Ontological dependence. Physical systems described by an EFT are ontologically dependent on physical systems described by a high-energy theory.

Claims (a) and (b) are suggested by the formal distinction between an effective Lagrangian density and a high-energy Lagrangian density, and their corresponding Euler-Lagrange equations of motion. In the case of (b), this suggests that the degrees of freedom of an EFT are dynamically distinct from those of a high-energy theory; moreover, the former are typically encoded in field variables that are formally distinct from those that encode the latter (i.e., different field variables appear in the Lagrangian densities of an EFT and a high-energy theory). On the other hand, the fact that the degrees of freedom of the former can be identified, via Steps (I) and (II) outlined above, as the low-energy degrees of freedom of the latter suggests (c): the physical systems described by an EFT do not completely "float free" of the physical systems described by a high-energy theory.

I'd now like to flesh out the above interpretation with two examples, and then extract a notion of emergence from it. The following examples are of a top-down EFT for a 2-dimensional quantum Hall liquid, and a bottom-up EFT for general relativity. 


\section{Example 1. A Top-Down EFT for a 2-dim Quantum Hall Liquid.}

The high-energy degrees of freedom of a quantum Hall liquid describe electrons moving in a 2dimensional conductor and coupled to external magnetic and Chern-Simons fields. This is described by a non-relativistic Lagrangian density,

$$
\mathcal{L}=i \psi^{\dagger}\left\{\partial_{t}-i e\left(A_{0}-a_{0}\right)\right\} \psi-(1 / 2 m) \psi^{\dagger}\left\{\partial_{i}+i e\left(A_{i}+a_{i}\right)\right\}^{2} \psi+\mu \psi^{\dagger} \psi+\vartheta \varepsilon^{\mu \nu \lambda} a_{\mu} \partial_{\nu} a_{\lambda}
$$

where the field variable $\psi$ encodes the electron degrees of freedom, the pair $\left(A_{0}, A_{i}\right), i=1,2$, encodes the degrees of freedom of an external magnetic field, $a_{\mu}(\mu=0,1,2)$ encodes the degrees of freedom of a Chern-Simons field, $\mu$ is the chemical potential, and the coefficient $\vartheta$ is chosen so that the electrons are coupled to an even number of "internal" magnetic fluxes, and hence refered to as "composite" electrons (Schakel 2008, pg. 349). Technically, this description entails that, in the presence of a strong external magnetic field, the electrons experience the quantum Hall effect. This occurs when the conductivity $\sigma$ of the system becomes quantized in units of $e^{2} / h$; i.e., $\sigma=v\left(e^{2} / h\right)$, where $v$ is called the "filling factor". The Integer Quantum Hall Effect (IQHE) occurs for integer values of $v$ and the Fractional Quantum Hall Effect (FQHE) occurs for values of $v$ given by simple fractions. Both the IQHE and the FQHE are characterized by incompressibility and disipationless transport, properties associated with superconductors. This suggests that these effects characterize a state of matter distinct from the conductor and refered to as a quantum Hall liquid. ${ }^{4}$

The properties of a quantum Hall liquid can be derived from the high-energy theory (3) by integrating out the electron degrees of freedom. The remaining degrees of freedom of the bulk liquid can then be identified with two Chern-Simons fields, $a_{\mu},\left(A_{\mu}+a_{\mu}\right)$, described by a "pure" Chern-Simons effective Lagrangian density,

$$
\mathcal{L}_{e f f}=\vartheta \varepsilon^{\mu \nu \lambda} a_{\mu} \partial_{\nu} a_{\lambda}+\vartheta^{\prime} \varepsilon^{\mu \nu \lambda}\left(A_{\mu}+a_{\mu}\right) \partial_{v}\left(A_{\lambda}+a_{\lambda}\right)
$$

where the coefficient on the last Chern-Simons term is chosen to produce the integer QHE for the second CS field (Schakel 2008,pg. 349). This is an example of a topological quantum field theory (i.e., a QFT encoded in a Lagrangian density in which a spacetime metric does not explicitly appear).

In this example, the high-energy Lagrangian density (3) is formally distinct from the effective Lagrangian density (4): (3) encodes a non-relativistic quantum field theory (QFT), whereas (4) encodes a topological QFT. This suggests that the laws of the EFT are not deducible consequences of the laws of the high-energy theory (failure of law-like deducibility); and that the EFT is dynamically distinct from the high-energy theory. Dynamical distinctness, coupled with the formal distinction between the field $\psi$ that encodes the degrees of freedom of the high-energy

\footnotetext{
${ }^{4}$ The IQHE can be explained by reference to the discrete spacing between the energy levels of the system. The filling factor is given by $v=$ (\#of electrons)/ (\# of states per energy level). At integer values of $v$, the first $v$ energy levels are full, and this entails incompressibility in the sense that no further electrons can be excited without a large cost in energy. The FQHE can be explained by noting that attaching an even number of fluxes to each electron cancels just enough of the external magnetic field to change the filling factor back to an integer value. Thus (in this description), the FQHE is the IQHE for composite electrons (Schakel 2008, pg. 343).
} 
theory and the fields $a_{\mu},\left(A_{\mu}+a_{\mu}\right)$ that encode the degrees of freedom of the EFT, suggest that the later characterizes physical systems (i.e., two topological Chern-Simons fields) that are ontologically distinct from those characterized by the former (i.e., non-relativistic composite electrons). Finally, the fact that the degrees of freedom of (4) are exactly the low-energy degrees of freedom of (3) suggests that the physical systems described by (4) are ontologically dependent on those characerized by (3). In particular, the bulk quantum Hall liquid characterized by the topological fields $a_{\mu},\left(A_{\mu}+a_{\mu}\right)$ ultimately consists of non-relativistic composite electrons.

\section{Example 2. A "Bottom-Up" EFT for General Relativity.}

Recall that a bottom-up EFT is constructed in the absence of a high-energy theory by first identifying the relevant symmetries of the phenomenon in question and then constructing an effective Lagrangian density as a local operator expansion (2) that includes all possible interactions consistent with these symmetries. In the case of general relativity, these symmetries are general covariance and local Lorentz invariance. If one assumes that the metric $g_{\mu \nu}$ encodes low-energy degrees of freedom of an unkown high-energy theory, then an effective Lagrangian density corresponding to (2) can be given by,

$$
\mathcal{L}_{\text {eff }}=\sqrt{g}\left\{\lambda+c_{1} R+c_{2} R^{2}+c_{3} R_{\mu v} R^{\mu v}+\ldots+\mathcal{L}_{\text {matter }}\right\}
$$

where $g=\operatorname{det}\left(g_{\mu \nu}\right), R, R_{\mu \nu}$ are the Ricci scalar and Ricci tensor, the $c_{i}$ are coupling constants, and the elipses refer to higher-order terms (Donoghue 1995, pg. 7). The Euler-Lagrange equations of motion generated by the first two terms are the Einstein equations with cosmological constant $\lambda$, and one can argue that the effect of higher-order terms is beyond current tests of general relativity.

In this example, since a high-energy theory is not known, the EFT is trivially characterized by the failure of law-like deducibility and ontological distinctness. Ontological dependence is secured by the assumption that the field variable $g_{\mu \nu}$ encodes the low-energy degrees of freedom of the unknown high-energy theory.

\section{Emergence in EFTs}

The philosophical literature typically distinguishes between two senses of emergence. The first views emergence as descriptive of the ontology (i.e., entities or properties) associated with a physical system with respect to another. To say phenomena associated with an EFT are emergent in this ontological sense is to say the entities or properties described by the EFT emerge from those described by a high-energy theory. A second sense of emergence views it as a formal relation between theories. To say phenomena associated with an EFT are emergent in this sense is to say the EFT stands in a certain formal relation to a high-energy theory.

Note that an EFT does not stand in a precise mathematical relation to a high-energy theory. As outlined in Section 2, Step (I) in the construction of an EFT requires both a choice of cutoff and a choice of low-energy degrees of freedom with respect to the latter. These choices typically will be dictated by the specific context of the problem at hand, as opposed to being products of a 
formal procedure. Similarly, the local operator expansion in Step (II) requires a context-specific identification of the symmetries of the high-energy theory (when it exists) or of the phenomena under investigation. This suggests that a purely formal concept of emergence for EFTs may not be appropriate. The approach adopted in this section will be to extract an ontological concept of emergence from the interpretation of EFTs suggested in Section 3. This interpretation motivates the following desiderata.

(i) First, the emergent system should ultimately be composed of microphysical systems that comprise the fundamental system and that obey the fundamental system's laws.

(ii) Second, the properties of the emergent system should not be deducible from the properties of the fundamental system.

I will follow Mainwood (2006, pg. 20) in refering to these desiderata as microphysicalism and novelty, respectively. They are underwritten in the EFT context by the elimination of degrees of freedom in the construction of an EFT. In particular, one might tell the following story about how the properties (and/or entities) of a system described by an EFT, encoded in an effective Lagrangian density $\mathcal{L}_{\text {eff }}$, emerge from a fundamental system described by a high-energy theory encoded in a Lagrangian density $\mathcal{L}$ :

(i) First, the high-energy degrees of freedom are identified and integrated out of $\mathcal{L}$. This entails that the degrees of freedom of $\mathcal{L}_{\text {eff }}$ are exactly the low-energy degrees of freedom of $\mathcal{L}$. Thus is microphysicalism secured.

(ii) Second, the elimination of degrees of freedom also entails that the solution $\mathcal{L}_{\text {eff }}$ of the path integral (1) is dynamically distinct from $\mathcal{L}$, and is a functional of field variables that do not appear in $\mathcal{L}$. Dynamical distinctness suggests a failure of law-like deducibility from $\mathcal{L}$ of the properties described by $\mathcal{L}_{\text {eff }}$, and a difference in field variables suggests the properties and entities described by $\mathcal{L}_{\text {eff }}$ and $\mathcal{L}$ are ontologically distinct. Thus is novelty secured.

\section{Other Notions of Emergence}

To further flesh out the above notion of emergence for EFTs, it will be helpful to compare it with other accounts in the philosophical literature.

\section{1. "New Emergentism", Spontaneous Symmetry Breaking, and Universality.}

Mainwood (2006, pg. 20) characterizes the "new emergentism" of prominent condensed matter physicists (e.g., Anderson 1972, Laughlin and Pines 2000) in terms of microphysicalism and novelty, as described above, underwritten by a physical mechanism. According to Mainwood, the specification of the latter is essential to avoid trivializing the concept of emergence:

"...emergent properties are not a panacea, to be appealed to whenever we are puzzled by the properties of large systems. In each case, we must produce a detailed physical mechanism for emergence, which rigorously explains the qualitative difference that we see with the microphysical" (pg. 284). Such a mechanism plays both an explanatory and a formal role. First, it explains how novelty arises: New Emergentists "...follow a strategy of first exhibiting 
evidence for emergence: the novel and unexpected character of certain systemic properties, and only then presenting a physical process - a 'mechanism' - that explains how such novelty can arise" (pg. 87). Second, formally, it underwrites the elimination of degrees of freedom from a constituative system, resulting in a system characterized by fewer degrees of freedom and exhibiting emergent phenomena. For Mainwood, the physical mechanism of most interest that accomplishes these tasks is spontaneous symmetry breaking (SSB): "The claim of the New Emergentists is that in the phenomenon of symmetry-breaking we have a mechanism by which the set of 'good coordinates' of the whole can be entirely different from the sets of good coordinates which apply to the constituent parts when in isolation or in other wholes" (pg. 107). However, Mainwood is careful to note that, in addition to SSB, the New Emergentists identify other mechanisms including renormalization, the integer and fractional quatum Hall effects, and localization (pg. 93), as well as universality (pg. 116).

SSB is the mechanism associated with the Landau-Ginzburg theory of phase changes in condensed matter systems, and its extension by renormalization group (RG) techniques. These theoretical frameworks associate phases with internal orders characterized by symmetries, and phase transitions with symmetry breaking. In the RG approach, phase transitions are analyzed by observing the behavior of a theory as its parameters are rescaled. Such rescaling generates a flow in the theory's abstract parameter space. A fixed point of such a flow is a point at which the values of the parameters remain unchanged under further rescaling; i.e., they become scale invariant. This occurs at a critical point corresponding to a phase transition. Thus phase transitions are characterized by scale independence: the properties associated with a phase transition are independent of the micro-scale properties of the system. In general, there can be many distinct RG flows that terminate at a given fixed point. A fixed point $x$ thus defines a universality class insofar as the theory defined by $x$ is independent of the microphysical details of any theory on an RG flow that terminates at $x$.

Both SSB and universality play essential roles in two other recent discussions of emergence in physics. These accounts view universality as underwriting the ontological non-reductivism they deem necessary in descriptions of emergent phenomena, but differ on the significance of SSB. On the one hand, Batterman (2011, pg. 1034) has suggested that the notion of a protectorate (i.e., a universality class) underwrites a concept of emergence "...that goes beyond mere claims to the effect that symmetry breaking occurs." According to Batterman (2011, pg. 1038), "It seems hardly satisfactory to appeal to symmetry breaking as an organizing principle independent of microdetails when we have such a profoundly successful story about why the microdetails in fact are largely independent or irrelevant." On the other hand, Morrison (2012,pg. 157) focuses explicitly on SSB as essential to the concept of emergence: "Although the RG provides an explanatory framework that shows why microphysical details can be ignored, it does not give us the kind of physical dynamics required for the production of emergent phenomena. For that we need symmetry breaking and the accompanying phase transitions". Morrison (2012, pg. 147) moreover suggests that "understanding emergent phenomena in terms of symmetry breaking -- a structural dynamical feature of physical systems... -- clarifies both how and why emergent phenoemena are independent of any specific configuration of their microphysical base." To support this claim, Morrison (2012, pp. 153-155) discusses an example due to Weinberg (1986) in which the essential properties of a superconductor are derived, not from a theory of its 
microconstituents (i.e., Cooper pairs), but by imposing symmetry constraints directly on a Lagrangian density.

Weinberg's example is instructive in the context of this essay insofar as it is an example of a bottom-up EFT. This raises two questions: First, how are SSB and universality related to EFTs, and second, if we agree with the above authors in their insistence on identifying a mechanism to underwrite a nontrivial concept of emergence, what is the nature of this mechanism in the EFT context?

The answer to the first question is explicit in the two examples discussed in Section 3: neither involves SSB or universality, at least as the latter is usually defined. Example 1 involves a phase transition from a less ordered conductor state to a more ordered quantum Hall liquid state; however, the orders cannot be distinguished by their symmetries. Wenn $(1995,2004)$ has developed a theory of "topological orders" that characterize the states associated with quantum Hall liquids, and argues that such liquids cannot be described by the standard Landau-Ginzburg theory of phase changes governed by SSB. ${ }^{5}$ Moreover, while quantum Hall liquids may be described in terms of a concept of universality, assumedly it will not involve the same technical description as that provided by the RG analysis of fixed points. ${ }^{6}$ In this broader sense, SSB is sufficient, but not necessary for universality. Example 2 also is not characterized by SSB or universality. In general, while the expansion point in the local operator expansion (2) of an effective Lagrangian density is defined by a fixed point (and hence a universality class) ${ }^{7}$, an EFT itself need not be identified with a fixed point, nor, necessarily, with a point on an RG flow that terminates at a fixed point. Both of the latter correspond to renormalizable theories, whereas EFTs in general need not be renormalizable. ${ }^{8}$ This suggests that a concept of emergence based on universality is too narrow for the EFT context. (It also suggests that a concept of emergence based on universality will have to include as emergent those phenomena associated with renormalizable theories; in particular, all the phenomena associated with the Standard Model would count as emergent.)

A concept of emergence appropriate for EFTs should thus be broader than a concept underwritten by SSB and/or universality. In Section 4 I suggested that emergence in EFTs be

\footnotetext{
${ }^{5}$ Wenn (1995, pg. 408) observes that the ground-state degeneracy that characterizes a quatum Hall liquid is not a consequence of the symmetry of the corresponding Hamiltonian, but rather depends on spatial topology. This fact, together with the fact that the ground-state degeneracy is robust under perturbations, suggests to Wenn that it be associated with a notion of universality characterized, not by symmetries and RG fixed points, but by topological order.

${ }^{6}$ Mainwood (2006, pg. 264, f.n. 3) acknowledges that the general concept of a universality class as used by New Emergentists "...is clearly meant to also extend beyond areas in which the RG techniques are usually applied".

${ }^{7}$ For weak interactions, the point of expansion $\mathcal{L}_{0}$ is taken to be a Gaussian fixed point in the parameter space of the high-energy theory, but any fixed point will serve this purpose. In general, the terms in the local operator expansion (2) are characterized by their behavior with respect to a fixed point (they can either increase, decrease, or remain the same as the RG flow approaches the fixed point), and this characterization is essential to the behavior of the EFT.

${ }^{8}$ A fixed point corresponds to a renormalized theory; i.e., a theory that is energy scale-independent. A point on an RG flow that terminates at a fixed point corresponds to a non-renormalized renormalizable theory; i.e., a "bare" theory that is capable of being made energy scale-independent, but whose parameters have not yet been rescaled to make this so. The most general form (2) of an EFT encompasses both of these theory types, but also a theory represented by a point on an RG flow that passes through a neighborhood of a fixed point, but does not intersect it. Such a theory is non-renormalizable.
} 
characterized in terms of microphysicalism and novelty, and that these characteristics are underwritten simply by the elimination of degrees of freedom in the construction of an EFT. Both Mainwood and Morrison require a causal/mechanical explanation of emergent phenomena in terms of a physical dynamical process like SSB (Batterman, on the other hand, is content with a unifying explanation based on the renormalization group). Morrison (2012, pg. 160), in particular, views an appeal to the elimination of degrees of freedom as not enough: "[t]he important issue...is not just the elimination of irrelevant degrees of freedom; rather it is the existence or emergence of cooperative behavior and the nature of the order parameter (associated with symmetry breaking) that characterizes the different kinds of systems." In response, I would agree that, by itself, an appeal to the elimination of degrees of freedom does not explain the existence of cooperative behavior, nor does it explain the existence and novel nature of emergent phenomena. On the other hand, the particular emergent phenomena associated with EFTs are not essentially characterized by cooperative behavior: while some examples are (quantum Hall liquids as described by EFTs), others are not (general relativity as described by an EFT). Moreover, within the interpretive framework suggested in Section 3, the elimination of degrees of freedom in an EFT does fulfill a causal/mechanistic explanatory role. In particular, the elimination of degrees of freedom in an EFT explains the existence and novel nature of lowenergy emergent phenomena by explaining how they are related to high-energy phenomena by a failure of law-like deducibility, and by ontological distinctness tempered by ontological dependence. Thus I would argue that the particular type of elimination of degrees of freedom in an EFT, coupled with an appropriate intepretation of EFTs, succeeds in doing the explanatory work deemed necessary by Mainwood and Morrison for a nontrivial concept of emergence.

\section{2. "Weak Ontological Emergence".}

An approach to a concept of emergence that stresses the importance of the elimination of degrees of freedom is given by Wilson (2010), who refers to this concept as "weak ontological emergence". 9 The elimination of degrees of freedom in a theory in physics, according to Wilson, involves the imposition of constraints that eliminate functional dependences between system properties and some subset of degrees of freedom (pg. 284). ${ }^{10}$ Wilson takes the following to be examples of this:

1. The electric field of a spherical conductor, which depends only on the degrees of freedom of the charges on its boundary (pp. 285-286).

2. Statistical mechanical aggregates: "[S] uccessful applications of the RG method to certain composed entities indicate that such entities have DOF [degrees of freedom] that are eliminated relative to systems consisting of their composing [parts]" (pg. 288).

3. Quantum degrees of freedom in the classical limit (pp. 288-290).

\footnotetext{
${ }^{9}$ Wilson (2010, pg. 280) takes "weak ontological emergence" to be compatible with physicalism, as opposed to "strong ontological emergence", which is not.

${ }^{10}$ Wilson (2010, pg. 282) considers a more general notion of a degree of freedom that the one adopted in Section 2, allowing that it need not necessarily figure into a state description that underwrites a dynamics.
} 
These examples arise in different contexts, none of which is appropriate for EFTs. Example 1 arises in the context of a single theory by the imposition of boundary conditions on the theory's equations of motion; thus it does not apply to the EFT context which involves two formally and dynamically distinct theories. Example 2 is drawn from discussions in Batterman (2002) and elsewhere and arises in the context of two theories (statistical mechanics and thermodynamics) related by a limiting relation. Arguably, this example also does not apply in general to the EFT context: Briefly, the procedure involved in constructing an EFT, as outlined in Section 2 above, does not produce a limiting relation between the EFT and its high-energy theory (see Bain 2012, pp. 28-32, for further discussion of Batterman's (2002) notion of emergence in the context of EFTs). Finally, Example 3 also seems to arise from an assumed limiting relation between two theories (classical and quantum mechanics), and thus is not applicable to EFTs. (The nature of the limiting relation in Example 3 is a bit more controversial than in Example 2, insofar as more than one dynamically distinct quantization of a given classical system can be constructed).

In the construction of an EFT, the elimination of degrees of freedom is not characterized by a limiting relation between theories, nor by the imposition of constraints on a set of equations of motion. Rather, it is characterized by the imposition of a constraint (in the form of a boundary condition that imposes an energy, or minimum length, cutoff) directly on the degrees of freedom of a Lagrangian density, as opposed to its equations of motion. This yields a formally distinct effective Lagrangian density with a distinct set of equations of motion. This formal distinctness severs functional dependences between the remaining low-energy degrees of freedom and the dynamics of the high-energy theory.

This type of elimination of degrees of freedom in an EFT does not appear to be what Wilson has in mind. Wilson takes the sort of elimination of degrees of freedom that underwrites ("weak ontological") emergence to play two roles. First, it establishes the physical acceptability of an emergent entity by securing the law-like deducibility of its behavior from its composing parts. This is taken to partially underwrite a concept of physicalism: ${ }^{11}$

...so long as a given special science treats only of entities $E$ whose characterization requires the same or fewer DOF [degrees of freedom] as their composing $e_{i}$, the special science is appropriately seen as extracted from the more fundamental science treating the $e_{i}$, such that the laws of the special science (expressing, in particular, the properties and behavior of $E$ ) are deducible consequences of the laws of the more fundamental science (expressing, in particular, the properties and behavior of the $e_{i}$ ). This is the case, in particular, with the special sciences (statistical and classical mechanics) treating entities satisfying Weak ontological emergence (Wilson 2010, pg. 295).

\footnotetext{
${ }^{11}$ For Wilson, physicalism in the context of weak ontological emergence is also underwritten by the claim that "...the law-governed properties and behavior of [an emergent entity] are completely determined by the law-governed properties and behavior of the [composing entities]..." (2010, pg. 280). If "completely determined" refers to an ontological notion of dependence between the emergent and fundamental entities, then this amounts to the notion of microphysicalism in Section 4. But if "completely determined" refers to a formal characteristic of a set of equations of motion, then I would argue that it is too strong a criterion on which to base a notion of physicalism. In particular, it fails in the context of typical EFTs.
} 
Second, according to Wilson, the elimination of degrees of freedom entails that an emergent entity is characterized by different law-governed properties and behavior than those of its composing parts. This is taken to underwrite a failure of ontological reductionism:

The line of thought appeals to the laws that scientists take to govern an entity of a given type, as providing an appropriate basis for identifying the DOF associated with that entity... [The argument] concludes that [the emergent entity] $E$ is not identical to [its composing parts] $e_{r}$, on grounds that there are scientific reasons for associating $E$ with certain laws, such that specifying $E$ 's law-governed properties and behavior requires certain DOF; and for associating $e_{r}$ with certain laws, such that specifying $e_{r}$ 's law-governed properties and behavior requires certain DOF different from those required to characterize $E$ (Wilson 2010, pg. 301).

This failure of ontological reductionism might charitably be associated with a notion of novelty, and this, coupled with physicalism might suggest a similarity between Wilson's weak onotological emergence and the sense of emergence in EFTs expounded in Section 4 above. However, again, the elimination of degrees of freedom that underwrites Wilson's physicalism and the failure of ontological reductionism is decidedly different from that which underwrites microphysicalism and novelty in EFTs: Where Wilson suggests elimination of degrees of freedom secures the law-like deducibility of an emergent entity from its composing parts, I've suggested that elimination of degrees of freedom in an EFT is characterized, in part, by a failure of law-like deducibility, and take this to underwrite novelty (in the sense of dynamical and ontological distinctness). I've also suggested that elimination of degrees of freedom in an EFT is also characterized by the retention, in the EFT, of the low-energy degrees of freedom of the highenergy theory, and it is this fact that underwrites a concept of (micro)physicalism (as opposed to a relation of law-like deducibility). Thus, while Wilson's concept of emergence may be applicable to some subset of physical systems described by theories in physics, it is not applicable to EFTs, under the interpretation suggested in Section 3.

\section{Conclusion}

This essay suggests that emergence in an EFT can be characterized by novelty and microphysicalism underwritten by the elimination of degrees of freedom from a high-energy theory. This is an elimination of degrees of freedom imposed directly on a high-energy Lagrangian density, as opposed to a set of equations of motion. It results in an effective Lagrangian density that can be interpreted as describing novel phenomena in the sense of being dynamically independent of, and thus not deducible from, the phenomena associated with a highenergy theory. These novel phenomena can be said to ultimately be composed of the phenomena that are constitutive of a high-energy theory, insofar as the degrees of freedom exhibited by the former are exactly the low-energy degrees of freedom exhibited by the latter. Finally it was argued in Section 5 that this concept of emergence in EFTs is more general than concepts of emergence based on spontaneous symmetry breaking and/or universality, but more narrow that a concept based simply on the elimination of degrees of freedom. 


\section{References}

Anderson, Philip. 1972. "More is Different." Science 177: 393-396.

Bain, Jonathan. 2012. "Effective Field Theories." Forthcoming in The Oxford Handbook of Philosophy of Physics, ed. Robert Batterman. Oxford: Oxford University Press.

Batterman, Robert. 2002. The Devil in the Details. Oxford: Oxford University Press. 1031-1050. . 2011. "Emergence, Singularities, and Symmetry Breaking." Foundations of Physics 41:

Donoghue, J. F. (1995). "Introduction to the Effective Field Theory Description of Gravity." arXiv:gr-qc/9512024v1.

Laughlin, Robert, and David Pines. 2000. "The Theory of Everything." Proceedings of the National Academy of Sciences 97: 28-31.

Mainwood, Paul. 2006. "Is More Different? Emergent Properties in Physics." PhD diss., University of Oxford. Online at PhilSci Archive: < http://philsci-archive.pitt.edu/8339>.

Morrison, Margaret. 2012. "Emergent Physics and Micro-Ontology". Philosophy of Science 79: 141-166.

Schakel, Adriaan. 2008. Boulevard of Broken Symmetries: Effective Field Theories of Condensed Matter. Singapore: World Scientific.

Wen, Xiao-Gang. 1995. "Topological Orders and Edge Excitations in Fractional Quantum Hall States". Advances in Physics 44: 405-473. . 2004. Quantum Field Theory of Many Body Systems. Oxford: Oxford University Press.

Weinberg, Steven. 1979. "Phenomenological Lagrangians." Physica 96A: 327-340. . 1986. "Superconductivity for Particular Theorists." Progress of Theoretical Physics Suppplement 86: 43-53.

Wilson, Jessica. 2010. "Non-reductive Physicalism and Degrees of Freedom." British Journal for Philosophy of Science 61: 279-311. 